\title{
High Level Darunavir Resistance in a Patient with HIV-1 RNA Rebound Under Antiretroviral Treatment
}

\author{
Müge TOYGAR DENIZ' ${ }^{1}$, Murat SAYAN ${ }^{2,3}$, S1la AKHAN ${ }^{1}$
}

\begin{abstract}
Today, antiretroviral therapies are successful in chronic HIV infection and have improved survival outcome. However antiretroviral drug resistance, coinfection, comorbidity and management of drug interactions are prominent in long-term treatments. In this study, we aimed to present the management of antiretroviral treatment in the light of high levels of darunavir resistance in a patient who is incompatible with antiretroviral therapies. HIV-1 drug resistance analysis was frequently applied because our patient was not compatible with the treatment. Minimally interacting agents with antiretroviral therapy could be selected in developing pulmonary tuberculosis. In addition, in the developing acute renal failure, genetic barrier anxiety could be left behind. Our findings suggest that HIV-1 drug resistance analysis should be an integral part of the management of antiretroviral treatment in developing co-infections and comorbidities in an incompatible patient.
\end{abstract}

Keywords: HIV, darunavir, protease inhibitors, drug resistance

\section{Introduction}

Chronic HIV infection continues to increase worldwide. According to the latest information from the Joint United Nations Program on HIV / AIDS (UNAIDS), 37.9 million people worldwide live with HIV (1). Although there is a relative increase of $20 \%$ in the total number of patients compared to 2010, the number of new HIV infections diagnosed as of the end of 2018 is 1.7 million and this

\footnotetext{
1 Kocaeli University Faculty of Medicine, Department of Clinical Microbiology and Infectious Diseases, Kocaeli, Turkey

Phone number: +90506 5856524 - +902623037084

e-mail:mugedeniz90@gmail.com

2 Kocaeli University, Faculty of Medicine, Clinical Laboratory, PCR Unit, Kocaeli, Turkey

3 Near East University, DESAM Institute, Nicosia, Northern Cyprus

DOI: 10.17932/EJOH.2020.022/ejoh_v01i1007
} 
number has decreased by $16 \%$ compared to 2010 . Similarly, by the end of 2018 , the annual rate of HIV-related deaths has been reduced by $33 \%$ compared to 2010 . This relative improvement in the HIV infection dynamics appears to have been achieved by increased access to antiretroviral therapy. According to the World Health Organization (WHO) data, today 23.3 million people living with HIV have access to antiretroviral treatment. According to estimation analysis, it is targeted to reach 33 million people by 2030 (2). On the other hand, in Turkey, there are 24,237 HIV-infected individuals confirmed as of July 2020 and approximately five people are infected with HIV-1 every day (3). According to other source, the International Pharmaceutical Market Database's (IMS) current data for Turkey (October 2019) 16,806 of our patients have access to antiretroviral treatment and 338 cases of the patients are under treatment with darunavir (11).

A combination treatment of two nucleoside reverse transcriptase inhibitor (NRTI) and non-nucleoside reverse transcriptase inhibitor (NNRTI), integrase inhibitor (INI) or enhanced protease inhibitor (PI) drug groups is recommended for an HIV-infected individual who has not previously received antiretroviral therapy (9). This combination treatment regimen is called combined antiretroviral therapy (cART), and studies have shown that HIV replication is suppressed and CD4 + $\mathrm{T}$ lymphocyte cell count increases when this form of treatment is started $(7,8)$.

In Turkey, the primary antiretroviral resistance ratio was determined to be $7.6 \%$ in patients infected with HIV. The distribution of resistance by drug groups is $4.2 \%$ in the NRTI group, $1.7 \%$ in the NNRTI group, and $1.7 \%$ in the PI group (12). However, a significant part of drug resistance emerges as acquired resistance due to inappropriate drug use.

Darunavir (DRV), a non-peptidic protease inhibitor, is effective on HIV protease by inhibiting enzymatic dimerization. DRV is an agent that is effective against PI resistant HIV strains both in vitro and in vivo, has a high genetic barrier, and resistance development is rare (5). In order to determine that the level of resistance to darunavir is high, at least four mutations must be present among the major mutations in the gene region encoding HIV protease (I50V / L, I54A / L / M / S / T / V, L76V, V82A / C / F / L / M / S / T, I84A / C / V, N88D/G/S/T, L90M in, N88D / G / S / T, L90M) (4).

This study aims to present the management of a case with a high level darunavir resistance seen in our country for the first time. 


\section{Case Report}

A twenty-five-year-old male patient was admitted to our hospital in 2009 after receiving positive results from the HIV antibody test before being operated on due to spontaneous pneumothorax. In routine tests, the patient's hemogram and biochemistry values were normal, CMV IgM negative, CMV IgG positive, toxoplasma IgM and IgG negative, anti-HCV, $\mathrm{HBs} \mathrm{Ag}$, anti-HBs, anti-HBc IgG negative, and anti HAV IgG positive serology result. Lymphocyte cell count was 98 cells / mm3, HIV-1 RNA load was 1,260,000 IU / ml. Antiretroviral drug resistance test before treatment showed the patient was sensitive to all drug groups. The patient was started on tenofovir / emtricitabine (TDF + FTC) + lopinavir / ritonavir (LPV/r) therapy and prophylaxis treatment for opportunistic infections.

Because the patient took medication irregularly, HIV-1 RNA did not become negative in May 2011, no resistance was detected to the NNRTI, NRTI and PI group drugs. HIV-1 drug resistance analysis was done by the Sanger dideoxy sequencing method. In the HIV-1 pol gene, the protease region (codon 1 to 99) and reverse transcriptase site (codon 1 to 235) were sequenced. In the resulting nucleotide sequences, mutations were identified in the Stanford HIVdb v8.7 program and clinical significance analyses were performed according to the World Health Organization-Transmitted Drug Resistance Mutation (WHO-TDRM) list (6). The treatments and drug resistance analyses performed since the beginning of patient follow-up are shown in Table 1. In addition, the patient's HIV-1 RNA load and CD4 + T lymphocyte cell count dynamics in the time series are shown in Figure 1.

In January 2018, antituberculosis treatment was initiated on the patient with positive asido-resistance bacilli (ARB) analysis in the sputum and compatible lung tomography. Antiretroviral therapy was administered as TDF / FTC + efavirenz (EFV) due to drug interaction. Because of a high resistance barrier and minimal drug interaction, the patient's treatment was arranged as TDF / FTC + dolutegravir (DTG) in May 2018. The patient's irregular drug use was ongoing at this time. In the control analyzes requested in the first month of this treatment, the number of CD4 + T lymphocyte cells was 33 cells / $\mathrm{mm}^{3}$, the HIV-1 RNA load was $3.440 .000 \mathrm{IU} / \mathrm{ml}$, and the concurrent resistance analysis showed sensitivity to zidovudine (ZDV), EFV, DRV agents and INI drug. The new treatment was arranged as DRV / $r+$ EFV due to sensitivity to the group.

In the HIV-1 drug resistance analysis done in November 2018, in the NRTI drug class, D67G, K70E gene mutations; in the NNRTI drug class, L100I, K103N gene mutations; in the PI drug class, V32I, M46I, I47V, V82A, I84V gene mutations; 
and in INI drug class, L33F, K43T, F53L gene mutations were detected. Upon determination of these mutations, it was determined that the NNRTI group was highly resistant, the PI group was highly resistant, and the NRTI group was considered to be abacavir, stavudine, didanosine-moderately resistant, tenofovir low-level, and ZDV sensitive. This is why the new treatment was arranged as $\mathrm{ZDV}+\mathrm{TDF} / \mathrm{FTC}+\mathrm{DTG}$.

In February 2019, as a result of abdominal tomography taken due to prolonged diarrhea, abdominal pain, vomiting and inability to urinate, a fistula extending from the anorectal junction to the bladder and right renal abscess as well as grade 2 hydronephrosis was detected. Operation was not considered by general surgery clinics and a bilateral nephrostomy was opened in the patient for postrenal acute renal failure. Treatment with meropenem and tigecycline antibiotherapy and antiretroviral therapy was changed to ZDV + tenofovir alafenamide / emtricitabine / cobicistat / elvitegravir (TAF / FTC / EVG / c) due to the high creatinine value $(2.67 \mathrm{mg} / \mathrm{dl})$. Under this treatment, the control HIV-1 RNA load was negative in August 2019, and the number of CD4 + T lymphocyte cells was determined as 60 cells / mm3. However, the general condition of the patient worsened and exitus was accepted due to intra-abdominal sepsis.

\section{Discussion}

DRV, which is an agent that is difficult to develop resistance to due to incompatibility in our case, was chosen, but during the 5th month of treatment, a high level of darunavir resistance was encountered. Disruption of a potent antiretroviral agent makes treatment management more difficult, although the treatment options are much more common today. On the other hand, with DTG, another agent with high genetic barrier, resistance mutations developed against the drug groups that the patient has previously used irregularly are suppressed. It should be remembered that HIV mutations are archived and can be detected in the plasma again as a result of drug group changes, even if they are sensitive to drug resistance analysis. In our case, the K103N mutation associated with EFV resistance was able to reappear long after treatment. On the other hand, the M184V mutation associated with the NRTI class is blamed for resistance to emtricitabine. However, despite the presence of a M184V mutation, when a potent antiretroviral is added from another drug class, M184V may not cause a problem (10). This detail can be valuable if there are limited options in patient management.

Increased viral load of our patient after one month of TDF / FTC + DTG caused drug changes to be made despite the high genetic barrier of DTG. However, the reason why the resistance test result turns out to be EFV sensitive is most likely 
because the high genetic barrier dolutegravir suppresses mutated strains in the plasma. Drug changes should be made by experts by evaluating the patient's previous drug history and resistance history together.

In the follow-up of our case as a result of being incompatible with treatment, HIV-1 drug resistance analysis was frequently used. In developing pulmonary tuberculosis, minimally interacting EFV and DTG agents could be selected with antiretroviral therapy, as well as genetic barrier anxiety could be left behind in developing acute renal failure. Our findings show that HIV-1 drug resistance analysis should be an integral part of antiretroviral therapy management in patients' co-infections and comorbidity.

\section{Conflict of Interest}

The authors did not report any conflicts of interest.

\section{References}

1. United Nations Joint Program on HIV / AIDS (UNAIDS) Data. [Internet]. (assessed date: 17 August 2020). https://www.unaids.org/en

2. World Health Organization, HIV / AIDS statistics. [Internet]. (assessed date: 21 November 2019). https://www.who.int/

3. T.C. Sağlık Bakanlığı, Bulaşıcı Hastalıklar Daire Başkanlığı. [Internet]. (assessed date: 20 November 2019). https://hsgm.saglik.gov.tr

4. Hoffmann C, Rockstroh JK, Kamps BS, editors. HIV Medicine 2007. 15th Ed. In:. Flying Publisher - Paris, Cagliari, Wuppertal.

5. Katlama C, Esposito R, Gatell JM, Goffard J-C, Grinsztejn B, Pozniak A, et al. Efficacy and safety of TMC114/ritonavir in treatment-experienced HIV patients: 24-week results of POWER 1. AIDS 2007; 21(4):395-402.

6. Sayan M, Sargin F, Inan D, Sevgi DY, Celikbas AK, Yasar K, et al. HIV-1 Transmitted drug resistance mutations in newly diagnosed antiretroviral naive patients in Turkey. AIDS Res Hum Retroviruses 2016;32(1):26-31

7. Moore RD, Bartlett JG. Dramatic decline in the HIV-1 RNA level over calendar time in a large urban HIV practice. Clin Infect Dis 2011;53(6):600-604.

8. Lee FJ, Amin J, Carr A. Efficacy of initial antiretroviral therapy for HIV-1 infection in adults: a systematic review and meta-analysis of 114 studies with up to 144 weeks' follow-up PLoS One 2014;9(5):e97482 
9. Guidelines for the use of antiretroviral agents in adults and adolescents with HIV (Last updated: October 2018). [Internet]. (assessed date: 23 November 2019) https://aidsinfo.nih.gov

10. Roger Paredes, Philip L. Tzou, Gert van Zyl, Geoff Barrow, Ricardo Camacho, Sergio Carmona, et al. Collaborative update of a rule-based expert system for HIV-1 genotypic resistance test interpretation PLoS One 2017; 12(7): e0181357.

11. İnternational Medical Statistics. [Internet]. (assessed date: 3 December 2019). https://www.sd.imshealth.com/tr/Pages/default.aspx

12. Sayan M, Sargın F, Inan D, Sevgi D Y,Çelikbaş A K, Yaşar K, et al. Transmitted antiretroviral drug resistance mutations in newly diagnosed HIV-1 positive patients in Turkey J Int AIDS Soc. 2014;17(4 Suppl 3):19750. 